\section{Monte Carlo simulation for microchannel plate framing camera}

\author{
Houzhi Cai, ${ }^{a}$ Jinyuan Liu, ${ }^{a}$ Lihong Niu, ${ }^{a}$ Wenda Peng, ${ }^{a}$ \\ Junlan Zhou, ${ }^{\mathrm{a}}$ Li Gu, ${ }^{\mathrm{a}}$ Hanben Niu, ${ }^{\mathrm{a}}$ and \\ Jinghua Long ${ }^{b}$ \\ aShenzhen University, Key Laboratory of Optoelectronics \\ Devices and Systems of Education Ministry, College \\ of Optoelectronic Engineering, Shenzhen 518060, China \\ bShenzhen University, College of Physics, Shenzhen \\ 518060, China \\ E-mail: ljy@szu.edu.cn
}

Abstract. A microchannel plate (MCP) framing camera is simulated by using the Monte Carlo method. Considering that the laser pulse width has effect on the experimental exposure time, we simulate the experimental exposure time varying with the laser pulse width. The gain characteristics of the MCP framing camera are simulated while the MCP is applied with very short electrical gating pulses. A new method is presented using a nongain MCP instead of a gain MCP. (๑) 2010 Society of Photo-Optical Instrumentation Engineers. [DOI: $10.1117 / 1.3479934]$

Subject terms: high-speed photography; framing camera; microchannel plate; Monte Carlo.

Paper 100320LRR received Apr. 16, 2010; revised manuscript received Jul. 17, 2010; accepted for publication Jul. 22, 2010; published online Aug. 19, 2010.

\section{Introduction}

A microchannel plate (MCP) framing camera is a powerful diagnostic tool for laser-driven inertial confinement fusion and fast Z-pinch experiments. ${ }^{1}$ Typically, exposure time of a framing camera gated by a MCP $0.5 \mathrm{~mm}$ thick is about 100 ps. The shortest exposure time of 35 ps was achieved by using a gated MCP $0.2 \mathrm{~mm}$ thick. $^{2}$

Theoretical simulation of the MCP framing camera has been widely studied. Eberhardt presented a discrete stage electron multiplier model for the MCP. He assumed that the stages of the electron multipliers were constant and independent of the applied voltage on the MCP. ${ }^{3}$ In this work, we assume that the stages are related to the applied voltage on the MCP.

Recently, characteristics of the electron cascade in a MCP are simulated based on the Monte Carlo method. ${ }^{4,5}$ Considering that the laser pulse width has effect on the experimental exposure time, we simulate the experimental exposure time varying with laser pulse width. Furthermore, the gain characteristics of the MCP are simulated, while the gating pulse width is not more than the electron transit time. A new method is presented using a nongain MCP instead of a gain MCP. The temporal characteristics of the nongain MCP framing camera are simulated.

\section{Microchannel Plate Model}

The MCP is an array of parallel continuous electron multipliers, assuming there is little influence between each chan-

0091-3286/2010/\$25.00 @ 2010 SPIE nel and all the channels have the same gain characteristics. Then, the electron cascade in the MCP can be approximated by the behavior of a single microchannel. ${ }^{4}$ The secondary electron yields, energies, and emission angles are the most important parameters in the simulation. The secondary electron yield is determined by the energy and incident angle of the primary electron. While the primary electron incident angle is $\theta$, assume that the primary electron with incident energy $V_{m}(\theta)$ will produce the maximum yield $\delta_{m}(\theta)$. The $V_{m}(\theta)$ and $\delta_{m}(\theta)$ are given as ${ }^{5}$

$V_{m}(\theta)=V_{m}(0) / \sqrt{\cos \theta}$,

$\delta_{m}(\theta)=\delta_{m}(0) \exp [\alpha(1-\cos \theta)]$,

where $V_{m}(0), \delta_{m}(0)$, and $\alpha$ are determined by the MCP lead glass. ${ }^{6}$ In our simulation, we take the data $V_{m}(0)=280 \mathrm{eV}$, $\delta_{m}(0)=4$, and $\alpha=0.5$.

The mean secondary emission yield for the primary electron with energy $V$ and incident angle $\theta$ is given as ${ }^{5}$

$\delta(V, \theta)=\delta_{m}(\theta) \cdot \frac{\frac{4 V}{V_{m}(\theta)}}{\left[1+\frac{V}{V_{m}(\theta)}\right]^{2}}$.

The actual secondary electron yield is acquired by random sampling from a Poisson distribution with the mean value given by Eq. (3).

The secondary electron energy is sampled randomly from a Maxwell probability distribution with the most probable energy of $2.5 \mathrm{eV}$. Energy conservation is considered in this work to ensure that the sum of the secondary electron emission energy from any given impact is less than the energy of the primary electron. The emission angle of each secondary electron is assigned by sampling from a cosine distribution, and the most probable angle of emission is along the surface normal to the channel wall.

The trajectory and velocity of each secondary electron can be calculated using the nonrelativistic equations of motion. Therefore, we can obtain the energy, incident angle, and position when the electrons strike the MCP channel wall. This impact process is repeated until the electrons emerge from the output surface of the MCP.

An ultraviolet (UV) laser is used to excite photoelectrons from the photocathode coated on the input surface of the MCP in the traditional exposure time measurement. ${ }^{2}$ While the MCP framing camera is operated in the gating state, a short electrical pulse is applied to the MCP. The exposure time is defined as the full width at half maximum (FWHM) of the MCP gain $G(t)$. From an experiment point of view, the $G(t)$ is hard to obtain due to the laser pulse width influence. We assume that the UV laser pulse is a temporal Gaussian distribution and converted to photoelectron pulse with the same distribution, given as

$n(t)=n_{p} \exp \left[-4 \ln 2\left(\frac{t}{\Delta t}\right)^{2}\right]$,

where $\Delta t$ is the laser pulse width, and $n_{p}$ is the amplitude of the Gaussian distribution set to be 100,000 . 


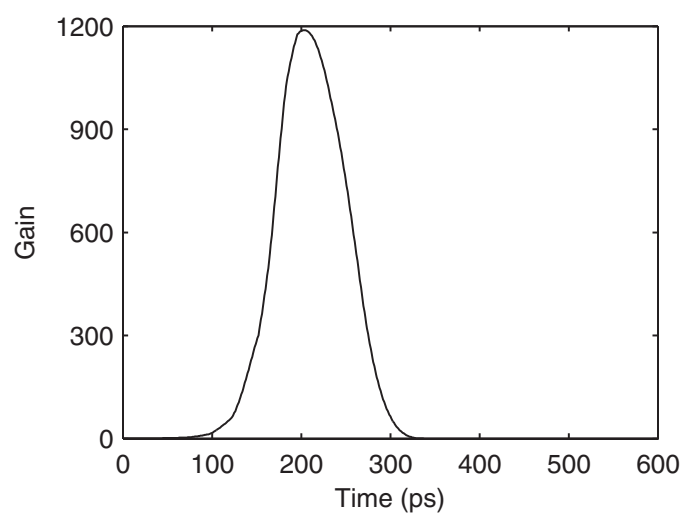

(a)

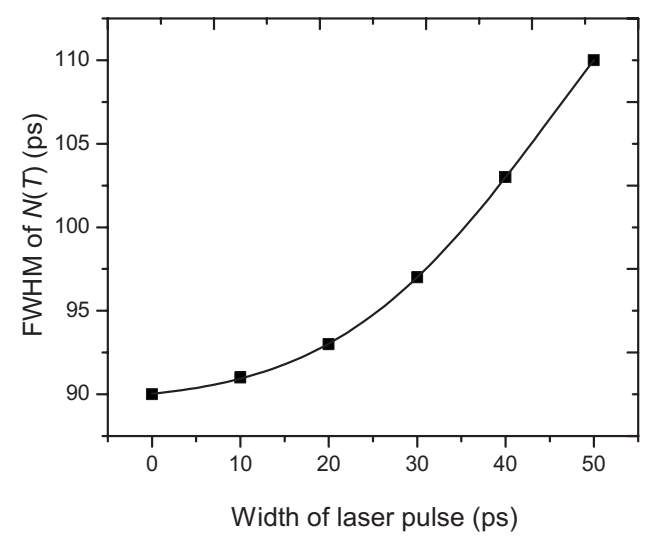

(b)

Fig. 1 Simulation results for a gating pulse with width of 300 ps and amplitude of $1 \mathrm{kV}$ applied to the MCP. (a) The gain curve $G(t)$ while the laser pulse width is zero. (b) Considering the laser pulse width, the FWHM of $N(T)$ versus the laser pulse width.

The Gaussian photoelectron pulse excited by the laser pulse at time $T$ is amplified by the MCP, and the number of electrons $N$ emerging from the channel exit can be deduced by

$N(T)=n(T) * G(T)=\int_{-\infty}^{+\infty} n(T-t) \cdot G(t) d t$.

It can be seen from the prior discussion that the measured result is the convolution between $n(T)$ and $G(T)$ in the traditional exposure time measurement.

\section{Simulation Results and Analysis}

MCP with channel diameter $d$ of $12 \mu \mathrm{m}$, thickness $L$ of $0.5 \mathrm{~mm}$, and bias angle $\theta$ of $6 \mathrm{deg}$ is simulated. The gating electrical pulse is a temporal Gaussian distribution the width of $W$ and amplitude of $V_{P}$, given as

$V(t)=V_{P} \exp \left[-4 \ln 2\left(\frac{t-W}{W}\right)^{2}\right]$.

The gating state simulation is initiated by introducing three electrons near the input end of the channel, typical of what might be expected from a $1-\mathrm{keV}$ x-ray. ${ }^{4}$ The introducing time for each of the three electrons range from 0 ps to $2 W \mathrm{ps}$, increased by a step of $0.1 \mathrm{ps}$. While the gating pulse

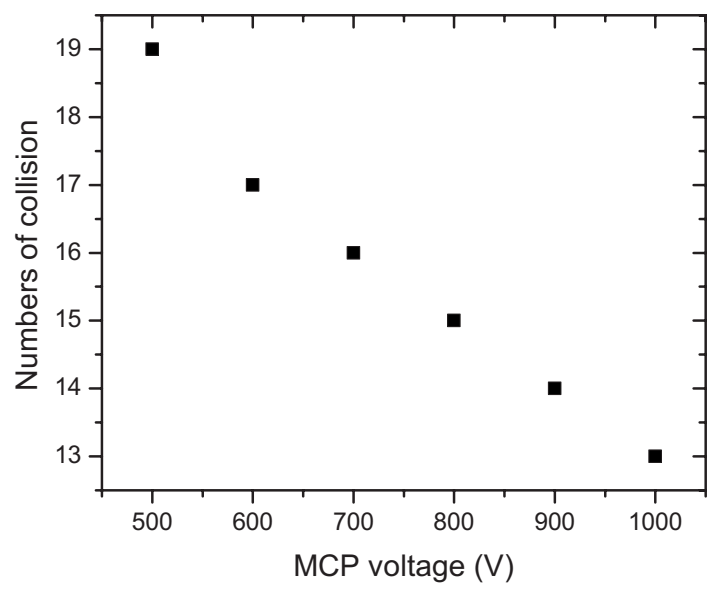

(a)

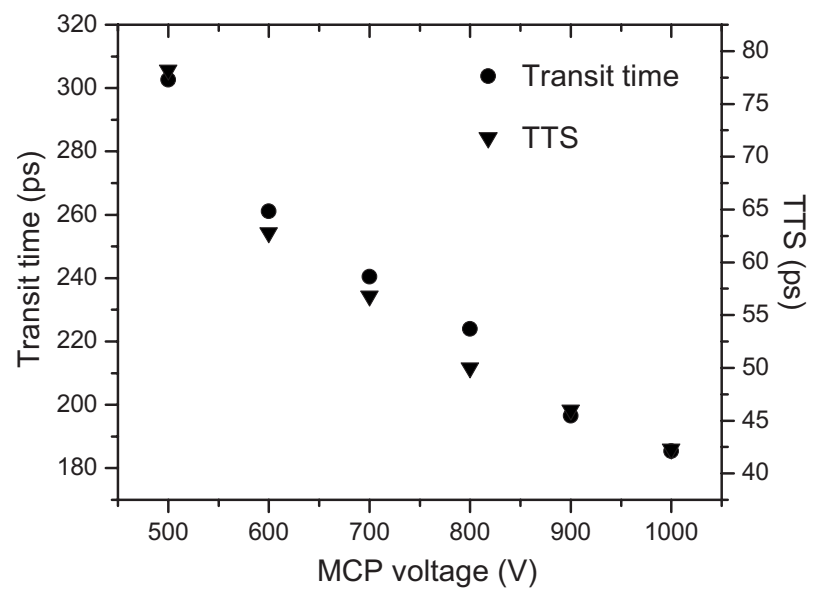

(b)

Fig. 2 Simulation results for different voltage applied to the MCP. (a) The collision numbers. (b) The transit time and TTS.

with a width of $300 \mathrm{ps}$ and an amplitude of $1 \mathrm{kV}$ is applied to the MCP microstrip line, the $G(t)$ can be obtained with Monte Carlo simulation. The gain curve $G(t)$ is plotted in Fig. 1(a). The FWHM of the $G(t)$ is 90 ps. It is the theoretical exposure time of the framing camera.

Since laser pulse width $\Delta t$ is given, $N(T)$ can be calculated using Eq. (5). Each curve $N(T)$ is calculated with laser pulse width $\Delta t$ ranging from 0 to $50 \mathrm{ps}$. The FWHM of curves $N(T)$ is presented in Fig. 1(b). It is clear from Fig. 1(b) that the laser pulse width strongly effects the exposure time measured result. If the laser pulse width is limited to zero, the measured result is close to the theoretical exposure time. Therefore, a laser pulse with larger width cannot be used in the exposure time measurement.

Figure 2 shows the collision numbers, the mean transit time, and the transit time spread (TTS) as a function of the direct current (dc) voltage applied to the MCP. The simulation is initiated by introducing single electron near the input end of the MCP. It can be seen from Fig. 2 that all the electron-channel wall collision numbers, the mean transit time of electrons traveling in the MCP, and the TTS are decreased while dc voltage is increased. While the acceleration is increased with increasing MCP voltage, the electrons travel a longer distance down the channel between 


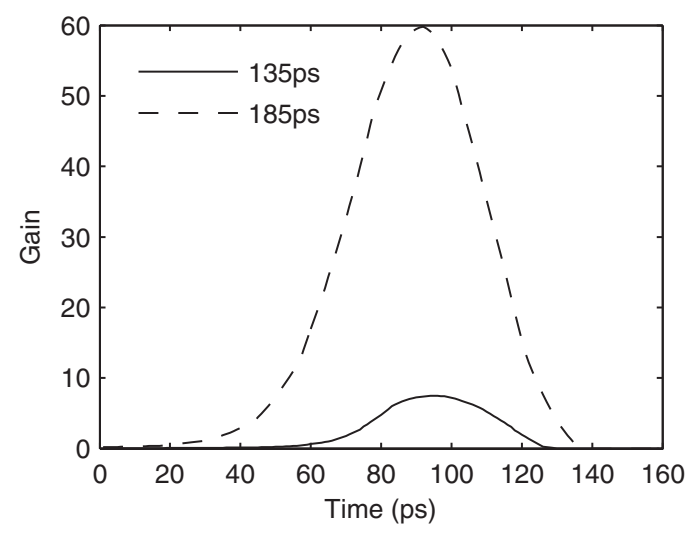

Fig. 3 The dynamic gain curves with different gating pulse widths: the broken line is the gain curve for the gating pulse with an amplitude of $1000 \mathrm{~V}$ and a width of $185 \mathrm{ps}$, and the solid line is that for the gating pulse with a width of $135 \mathrm{ps}$.

collisions, and that leads to a decrease of collision numbers. These factors result in decreasing the mean transit time and the TTS. As the dc voltage is $-1 \mathrm{kV}$, the electron transit time is $185 \mathrm{ps}$ and the TTS is $42 \mathrm{ps}$. These simulation results are consistent with existing measurements. ${ }^{5}$

Figure 3 shows the $G(t)$ with different gating pulse widths. The amplitude of the gating pulse applied to the $\mathrm{MCP}$ is $1 \mathrm{kV}$, and the widths are 185 and $135 \mathrm{ps}$, respectively. While the gating pulse width is $185 \mathrm{ps}$, FWHM of the gain curve is $45 \mathrm{ps}$ and maximum gain is 60 . While the gating pulse width is $135 \mathrm{ps}$, the maximum gain is less than 10 and the FWHM of gain curve is 40 ps. One can make a conclusion from Fig. 2(b) and Fig. 3 that if the gating pulse width is less than the electron transit time, the exposure time of the framing camera is approximately equal to the TTS, and the gain is low. The minimum exposure time is considered to be equal to the TTS. Therefore, although the gating pulse width is much shorter than the transit time, the exposure time cannot be shortened.

To shorten the exposure time, we present a new method using a nongain MCP instead of a gain MCP. The nongain MCP is an array of parallel channels. There is no material coated on the channel wall for secondary electron emission, so that the electrons impacting the wall will be absorbed. Only those photoelectrons with appropriate energy and emission angles can emerge from the output end of the channel without collision. The structure of the nongain MCP framing camera is the same as the previous MCP gated framing camera. ${ }^{1}$ The simulations of the nongain MCP are started by assuming some number of initial photoelectrons near the input end of the MCP. The photoelectron energy is sampled randomly from a Maxwell probability distribution, and the emission angle of each photoelectron is assigned by sampling from a cosine distribution. The trajectory of each photoelectron can be calculated using the nonrelativistic equations of motion. If the photoelectron strikes the MCP channel wall, it will be absorbed. Otherwise, it can emerge from the channel exit. The transit time and the TTS are plotted versus the applied voltage on the MCP in Fig. 4. The mean transit time and the

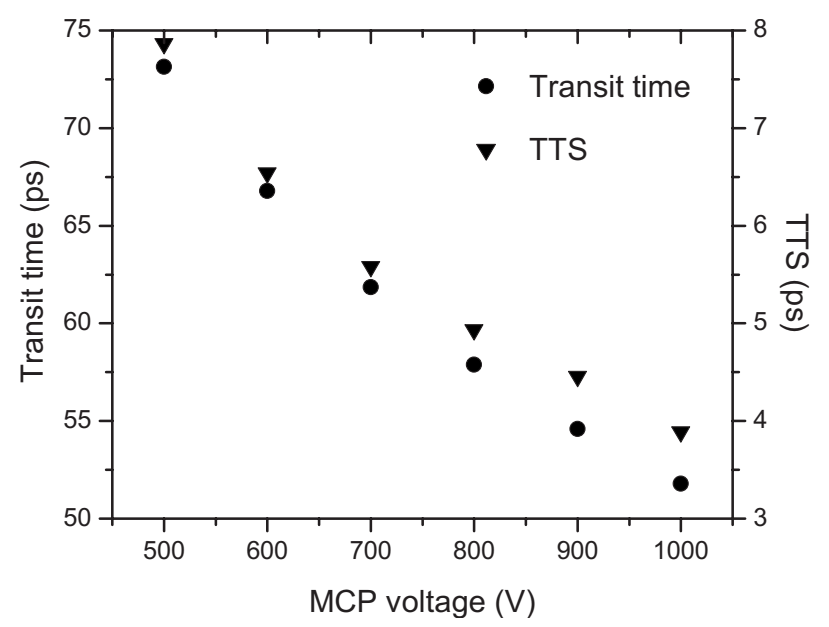

Fig. 4 The transit time and the TTS versus dc voltage in the nongain MCP.

TTS of the nongain MCP are decreased with the dc voltage increase. The TTS in the nongain MCP is one order of magnitude lower than that in the gain MCP. The exposure time in the nongain MCP framing camera is greatly shortened.

\section{Conclusions}

Simulation for a MCP framing camera based on the Monte Carlo method is described. The simulated results show that the exposure time measurement error is increased with the laser pulse width increasing in the exposure time measurement. A laser pulse width far less than the FWHM of $G(t)$ should be used in the experiment. The minimum exposure time is considered to be equal to the TTS. To shorten the exposure time, a new method is presented using a nongain MCP instead of the gain MCP. The TTS in the nongain MCP is much less than that in the gain MCP from the simulation results. Our future work is to measure the temporal resolution of the nongain MCP framing camera.

\section{Acknowledgment}

This work is supported by the Nature Science Foundation of China under grant number 10875080.

\section{References}

1. J. Liu, L. Niu, W. Peng, et al., "Application of a fast electrical pulse in gated multichannel plate camera," Rev. Sci. Instrum. 78(5), 055104 (2007).

2. P. M. Bell, J. D. Kilkenny, R. Hanks, and O. Landen, "Measurements with a 35 psec gate time microchannel plate camera," Proc. SPIE 1346, 456-464 (1990).

3. E. H. Eberhardt, "Gain model for microchannel plates," Appl. Opt. 18(9), 1418-1423 (1979).

4. M. Wu, C. A. Kruschwitz, D. V. Morgan, and J. Morgan, "Monte Carlo simulations of microchannel plate detectors. I. Steady-state voltage bias results," Rev. Sci. Instrum. 79(7), 073104 (2008).

5. M. Ito, H. Kume, and K. Oba, "Computer analysis of the timing properties in micro channel plate photomultiplier tubes," IEEE Trans. Nucl. Sci. NS-31(1), 408-412 (1984).

6. A. Authinaraynan and R. W. Dudding, "Changes in secondary electron yield from reduced lead glasses," Adv. Electron. Electron Phys. 40A, 167 (1976) 